$\Phi=$ 离

\title{
Spinal tuberculosis in a case of tubercular meningitis with hydrocephalus
}

\author{
Shriram Gautam ${ }^{1 *}$, Vinod K. S. Gautam ${ }^{2}$ \\ ${ }^{1}$ Junior Resident, Department of Neurosurgery, Institute of Human Behaviour and Allied Sciences, \\ Government of NCT Delhi, Dilshad Gardan, Delhi-110095, India \\ ${ }^{2}$ Assistant Professor, Department of Neurosurgery, Institute of Human Behaviour and Allied Sciences, \\ Government of NCT Delhi, Dilshad Gardan, Delhi-110095, India \\ *Corresponding author E-mail: shriram.lax@gmail.com
}

\begin{abstract}
Delayed diagnosis of tuberculosis of the brain and spine is associated with morbidity and mortality. Authors present a case to highlight the significance of thorough clinical work up, neuro-radiology and appropriate and prompt anti-tuberculous therapy in patients of CNS TB. Timely neurosurgical interventions in this patient who had developed spinal tuberculosis during the course of treatment for tuberculous meningitis (TBM) helped in reducing the morbidity and led to complete neurological recovery.
\end{abstract}

Keywords: CNS; Spinal Tuberculosis.

\section{Introduction}

Central Nervous System Tuberculosis (CNS TB) is caused by Mycobacterium tuberculosis, an obligate aerobic gram positive and acid fast bacillus (AFB). CNS TB comprises of approximately 10 percent of extrapulmonary tuberculosis cases and 1 percent of all TB cases [Gautam et al 2013].

Involvement of spine occur either secondary to Pott's spine or as non-osseous spinal cord tuberculosis. Vertebral body tuberculosis with cord impingement holds for the majority of cases with spinal involvement and most often presents with pain, gibbus and signs of extrinsic cord compression [Gautam et al 2015]. Non-osseous spinal tuberculosis includes spinal arachnoiditis, tubercular myelitis or tuberculomas.

\section{Case report}

A 48 year old female, resident of Delhi was admitted in Department of Neurosurgery in Institute of Human Behavior \& Allied Sciences (IHBAS), tertiary care Neuropsychiatry hospital, Delhi. She had initially presented with high grade intermittent fever, headache, altered sensorium and urinary retention. After neurological examination, neuroradiological evaluation and CSF study she was diagnosed as TBM with ostructive hydrocephalus. She was operated for Ventriculoperitoneal shunt insertion (VP shunt) by the author and a right sided medium pressure shunt was placed [figure 1]. Patient was kept on 4 drug anti-tubercular teratment (ATT) regimen consisting of Isoniazid, Rifampicin, Pyrazinamide, Ethambutol [HRZE]. Patient showed significant clinical improvement following the VP shunt surgery but bladder sensation did not return and patient remained catheterized with indwelling Foley's catheter.

At sixth month of follow up while on regular ATT, she started complaining of pain in upper back and band like sensation over the trunk. On neurological examination patient was found to have MRC grade 4/5 Para paresis with about 20 percent sensory loss below umbilicus with bladder involvement, which raised the suspicion of spinal TB. Skiagram of dorsal spine (figure 2) showed diminution of disc space between D8 and D9 vertebrae. MRI of dorsolumbar spine [figure 3A, B \& C] revealed paradiscal and epidural hypointense lesion on $\mathrm{T} 1$ weighted image which showed hyperintensity on T2 weighted image at D8-D9 vertebral level suggestive of tubercular granulation tissue.

Patient had no contact history of tuberculosis. There was no evidence of any systemic or pulmonary tubercular involvement except CNS. There was no lymphadenopathy and on hemogram there was no rise in erythrocyte sedimentation rate (ESR). Based on neuroradiological and clinical evaluation, streptomycin was added to the four drug regimen.

So, patient was operated by author and D8 and D9 laminectomy and excision of granulation tissue was done under general anesthesia and epidural granulation tissue was sent for histopathological examination. Patient improved post operatively with improved sensory deficit and decreased stiffness in lower limbs in immediate post-operative period. Patient regained motor power in the lower limbs and she started walking without any ambulatory support. However, patient continued with complain of urinary retention and abdominal pain. She was catheterized since 7 months. Her catheter continued to get blocked in couple of days with some pus like discharge in the tube. Oral Bethanechol $25 \mathrm{mg}$ 8 three times a day, stimulates parasympathetic receptors to increase bladder muscle tone, which in turn causes contraction and stimulates micturition was started for the urinary retention, patient responded well to the drug and urinary retention was subsided. Patient still complained of diminished sensation of fullness of bladder. USG abdomen shows signs of cystitis probably because of prolonged catheterization. Patient also passed urine with some stones of variable sizes after the administration of bethanechol. Stones were probably formed due to prolonged stasis of urine. Therefore, one should also look for stones and signs of cystitis in 
patients with prolonged catheterization due to bladder involvement due to of Pott's paraplegia.

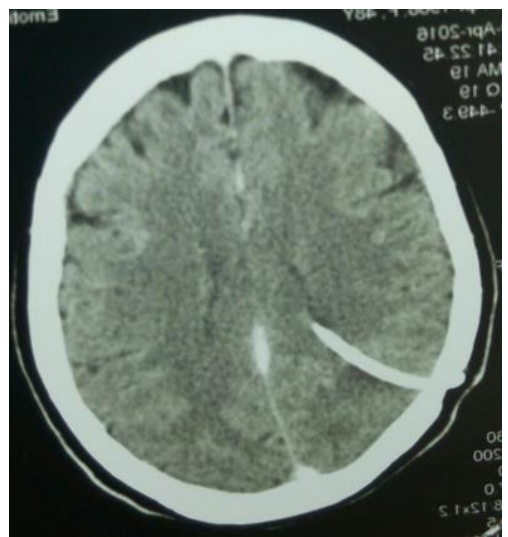

Fig. 1: Contrast Enhanced CT scan of the Head- Axial View Showing Ventricular End f VP Shunt in Situ with No Evidence of Hydrocephalus.

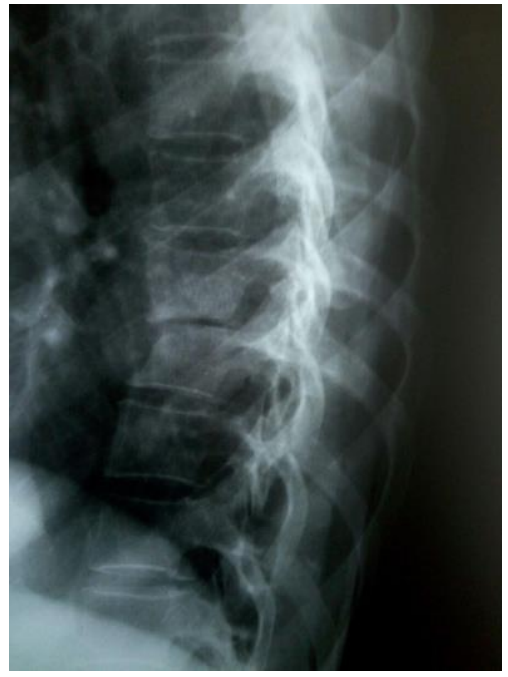

Fig. 2: Skiagram of Dorsolumbar Spine- Lateral View Showing Loss of Intervertebral Disc Space at the Level of D8-D9.

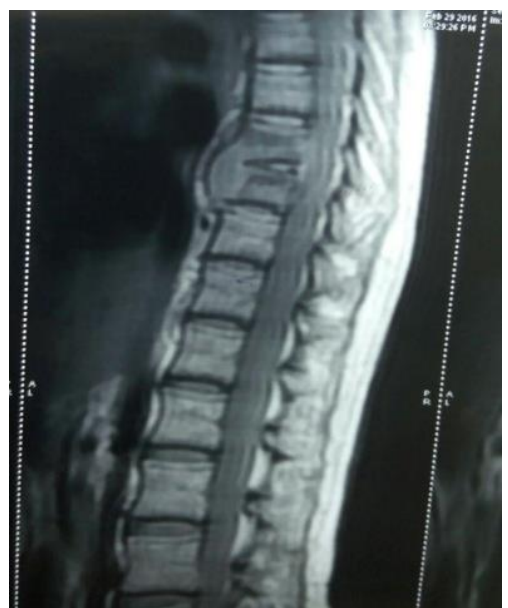

Fig. 3A: T1W1 Weighted MRI Image Sagittal View of Dorsolumbar Spine Showing Destruction of Vertebral Body and Granulation Tissue at D8-9 Vertebral Body.

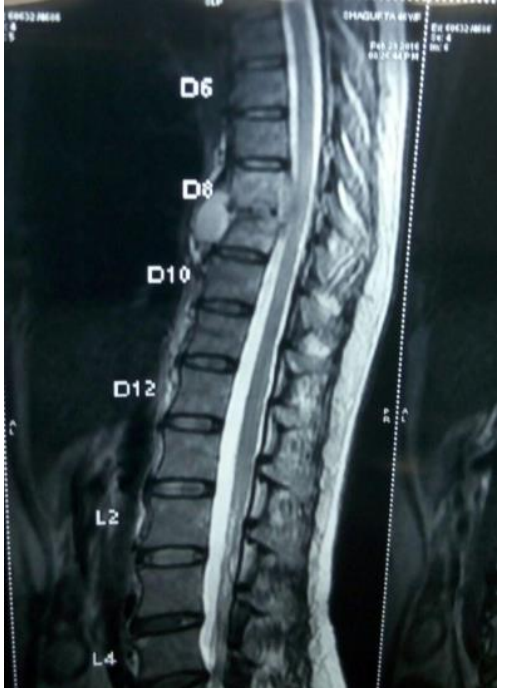

Fig. 3B: T2 Weighted MRI Image Saggital View of Dorsolumbar Spine Showing Hyperintense Granulation Tissue at D8-9 Vertebral Body and Compression of Spinal Cord.

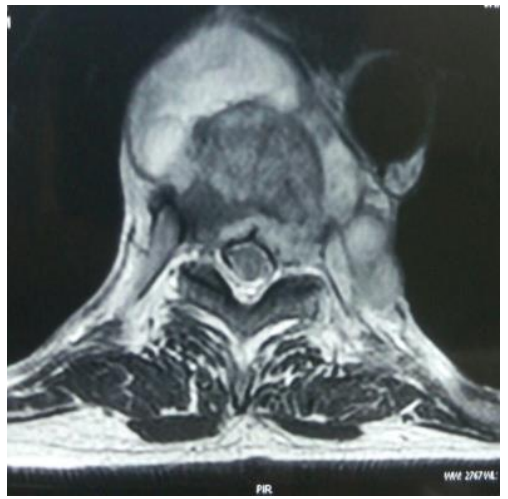

Fig. 3C: T2 Weighted Image of MRI Dorsolumbar Spine Axial View Reflecting Destruction of Vertebral Body and Hyperintense Granulation Tissue in Epidural Space and Anterior to Vertebral Body.

\section{Discussion}

While taking history and examining the patient some important points came to light that any intracranial tuberculosis case should be listened enthusiastically and examined efficiently to look for any evidence of spine or any other organ involvement concomitantly. Even after various diagnostic modalities and number of antitubercular drugs available for the treatment of TB clinician face difficulty in managing tubercular infection of CNS [Gautam et al 2013]. Regular follow up and close watch on response of drug is done by clinical examination and radiological methods in order to ensure effective response of drug. Prompt and timely decision of testing drug susceptibility is mandatory for converting $1^{\text {st }}$ line ATT to $2^{\text {nd }}$ line [Gautam et al 2015].

Involvement of spine occurs in less than $1 \%$ of TB patient and it can be secondary to Pott's spine or as non-osseous spinal cord tuberculosis. The thoracic spine is involved in about $65 \%$ of cases. [Gautam et al 2013].

Vertebral tuberculosis is the commonest form of skeletal tuberculosis, most series reporting an incidence of up to $50 \%$ of osteoarticular tuberculosis. Tuli reported that out of a total of 1,074 cases of osteoarticular tuberculosis, 440 cases affected the spine. Spinal tuberculosis can occur at any age and affects both sexes equally [Sridhar 2012, Tuli 1991]. Diagnosing a Pott's spine is a puzzling task and misdiagnosis is not uncommon, contributing to the development of potentially severe neurologic deficits. It is essential to have a high index of suspicion in such cases [Park et al 2006]. Spinal TB (Pott's or tuberculous spondylitis) often involves two or more adjacent vertebral bodies. From the anterior superior or inferior angle of the vertebral body, 
the lesion slowly reaches the adjacent body, later affecting the intervertebral disk. With advanced disease, collapse of vertebral bodies results in gibbus and kyphosis A paravertebral "cold" abscess may also form. In the upper spine, this abscess may track to and penetrate the chest wall, presenting as a soft tissue mass; in the lower spine, it may reach the inguinal ligaments or present as a psoas abscess. CT or MRI reveals the characteristic lesion and suggests its etiology [Kasper et al 2015].

The rapid and accurate diagnosis of symptomatic patients is the cornerstone of global strategies for tuberculosis (TB) control While progress in TB diagnostics has been made in developed countries, in the rest of the world the techniques used for diagnosing TB have remained relatively unchanged for the past few decades. These old tools are simply too slow and cumbersome, while the newer tools are still expensive and too complex. For countries sinking under TB's load, inadequate access to good diagnostics at all health service levels leaves many patients undiagnosed [WHO 2006]. Therefore, one should perform a thorough neurological examination, despite known previous diagnosis or images available, this will help us to find, what might have been missed or anything new has started. Take small repetitive history during treatment to get the complete sum of history, which, one has missed at initial history or the patient have recalled something clinically relevant. Nutritional and psychological support is required to build the strength and will in the patient, because a stressed and depressed and non-motivated patient may show less signs of improvement, a little motivation can bring surprising hidden improvements.

The spinal disease is always secondary to a primary lesion, usually from a visceral focus, and occurs due to haematogenous spread. Involvement of different parts of the spine and the presence of associated visceral lesions imply a bacillaemia causing the spread of infection from the primary focus. Infection may reach the spine due to a bacillaemia or through the Batson's plexus of veins. The primary focus may be active or quiescent and may be in the lungs, mediastinal lymph nodes, kidneys or other viscera. Simultaneous involvement of the paradiscal part of two contiguous vertebrae suggests spread of infection via the common blood supply to the region. On an aveyrage, an involvement of 3.4 vertebrae was reported in two series[Hodgson, Stock 1960, Martin 1970] 3.8 in another [Mukhopadhyaya , Mishra 1987] of non-contiguous multilevel involvement to be as high as $71.4 \%$ when screened with whole spine magnetic resonance imaging (MRI). [Sridhar K 2012, Kaila et al 2007].

Classically, four types of involvement of the spinal cord have been described in spinal tuberculosis: (i) a paradiscal lesion which arises from arterial spread of the infection (ii) the central type of vertebral body involvement of one or more distant or adjacent vertebrae this is often associated with tuberculosis meningitis as the spread of the infection is via the Batson's plexus of veins); (iii) the anterior type with cortical bone destruction and (iv) appendiceal type. [Sridhar K 2012, Tandon 1980]

Tuberculosis spine is frequently associated with disabling spinal cord and radicular complications. Available treatment options are far from satisfactory. It is difficult to assess the therapeutic response in the early follow up period in view of the lack of sensitive and specific tests. Vigilant clinical observation and imaging studies are required to identify the worsening or new emerging signs in the patient of CNS TB. Imaging studies are becoming the major decisive tool for the empirical therapy and early follow up of the patients to evaluate the therapy [ Gautam et al 2013]. Early diagnosis and treatment of spinal tuberculosis is essential to prevent complications like neurological deficit. The "gold standard" for diagnosis should ideally be isolation and culture of tuberculous bacilli. However, culture methods are slow and insensitive especially in cases of paucibacillary skeletal lesions [Shaikh et al 2013].

TB of CNS requires longer therapy preferably for one year. For any new casse of CNS TB, it is advised that the initiation phase (IP) of two months should contain four drugs (HRZE) and continuation phase should contain two drugs (HR). Continuation phase containing three drugs (HRE) as a daily regimen should be given only in countries with high levels isoniazid resistance in new TB patient, and where isoniazid drug susceptibility testing in new patient is not done (or results are unavailable) before the continuation phase is begins. Adjuvant corticosteroid treatment (Dexamethasone or Prednisolone) should be given at least for one month in severe cases of CNS TB [Gautam et al 2015].

\section{Conclusion}

The aims of Neurosurgical intervention in CNS TB patients are to confirm diagnosis, achieve bacteriological cure, prevent and/or treat deformity and neural compression. With technological advancements in the field of spinal imaging, early diagnosis of spinal tuberculosis is possible. With better chemotherapeutic drugs available that reach pus, granulation, caseous material and bone, non-operative management has improved, especially in cases diagnosed early. It is now recommended that radical excision is not necessary for infective spinal lesions, like tuberculosis, where effective anti-tubercular chemotherapy is available [Sridhar $\mathrm{K}$, 2012] .The treatment response is better with excision with granulation tissue and pus in surgically accessible areas of brain and spine.

\section{References}

[1] Gautam V.K.S, Khurana S, Singh. R.Diagnostic and therapeutic challenges in the surgical management of CNS Tuberculosis. International Journal of Medical and Health Sciences, April 2013 , Vol 2; Issue 2, 161-169.

[2] Gautam V.K.S, Khurana S, Singh. R. Multidrug resistant (MDR) tuberculous meningitis with hydrocephalus treated with ventriculoperitoneal shunting. International Journal of Medical and Health Sciences, 2015, 2(1), 22-25.

[3] Sridhar K. Tuberculosis of the Spine. Chapter in Ramamurthi and Tandon's Textbook of Neurosurgery, 3rd Ed, 2012, Jaypee New Delhi.

[4] Tuli S M. Tuberculosis of the Skeletal System. Jaypee Brothers Medical Publishers (P) Ltd.; 1991. New Delhi

[5] P Park, K Lewandrowski, R McLain. Pott's disease Associated with Tuberculous Meningitis Causing Blindness. The International Journal of spine surgery 2006, Vol 3 Number 2, 1-7 http://ispub.com/IJSS/3/2/3271.

[6] Kasper D.L., Hauser S.L, Jameson J.L, Fauci A.S., Longo D.L, Loscalzo J.Harrison's Principles of Internal Medicine (19th Ed) (Vols 1 page 1110) Mc Graw Hill ISBN: 978-0-07-180216-1

[7] Diagnostics for tuberculosis: global demand and market potential. World Health Organization publication 2006. www.who.int/tdr/publications/tbdi

[8] Hodgson AR, Stock FE. Anterior fusion for the treatmentof tuberculosis of the spine. J BoneJoint Surg. 1960; 42A:295-310.

[9] Martin NS. Tuberculosis of the spine-A study of the results of treatment during the last twenty-five years. J Bone Joint Surg. 1970 52B:613-28.

[10] Mukhopadhyaya B, Mishra IVK. Tuberculosis of the spine.Ind J Surg. 1987; 19:59.

[11] Kaila R, Malhi Am, Mahmood B, et al. The incidence of multilevel noncontiguous vertebral tuberculosis detected during whole spine MRI. J Spinal Disord Tech. 2007; 20:78-81. http://dx.doi.org/10.1097/01.bsd.0000211250.82823.0f.

[12] Tandon PN. Spinal tuberculosis. In: Ramamurthi B,Tandon PN (Eds). Textbook of Neurosurgery. New Delhi: National Book Trust; 1980. pp. 718-76.

[13] Sadik I Shaikh, Asma A Chauhan, Upendra Patel. An analysis of clinico-radiological and histopathological correlation in tuberculosis of spine. National journal of medical research Volume 3 | Issue 3 | July - Sept 2013. ISSN: 22494995. 